\title{
Translation Invariant Features from Cascaded Wavelet and Fourier Transforms for Bearing Fault Pattern Recognition Using Artificial Neural Network
}

\author{
Muhammad Amar, Iqbal Gondal, Campbell Wilson, and Ahmet Sekercioglu
}

\begin{abstract}
Rotary machine fault classification from vibrations requires robust feature extraction and enhancement procedures for transient and steady-state fault signatures. Accurate fault pattern classification relies on the quality of features extracted from the fault patterns. Fourier transform (FT) and wavelet transform (WT) based methods have largely been used for extraction of these features. FT performs well with non-stationary vibrations to provide translation invariant spectral features which can readily be used as input for classifier but belittles the spectral amplitudes of time-domain transients because of unmatched window size. WT, in contrast, deals well with transient's amplitude calculations from non-stationary vibrations because of signal decomposition into several frequency sub-bands but lacks in readily providing translation invariant features. As WT can better augment features and FT can readily provide translation-invariant spectral features suited for artificial neural network (ANN) classifier, therefore, this paper proposes a cascaded WT and FT based features extraction method for improved fault pattern recognition. The efficacy of proposed work is evaluated by comparing with existing methods. The results, under very poor SNR of -10dB, show that cascaded WT and FT based augmented and translation invariant features with ANN surpasses existing methods in classification accuracy under given conditions.
\end{abstract}

Index Terms-Artificial neural network (ANN), bearing fault, machine health monitoring (MHM), multi resolution analysis (MRA).

\section{INTRODUCTION}

There are several causes of mechanical faults in rotary machines and vibration signals are the indicative of the majority of these faults. Therefore, vibration signals are among the best candidate for bearing faults and other machine health monitoring (MHM) purposes [1]. In a most common approach, as a first step, the vibrations are recorded and time segmented using a rectangular window. Depending upon the nature of the fault, duration of fault signatures varies from very short, transients, to very large, steady-state, relative to time segmented window length. These, time segmented vibrations are used to extract features for the training and testing of the classifier for fault classification [2] Altogether, fault patterns classification is linked with robust

Manuscript received May 28, 2014; revised October 24, 2014.

M. Amar and C. Wilson are with Faculty of Information Technology, Monash University, Australia (e-mail: muhammad.amar@monash.edu, campbell.wilson@monash.edu).

I. Gondal is with Monash University and Federation University, Australia (e-mail: iqbal.gondal@federation.edu.au).

A. Sekercioglu is with the Department of Electrical and Computer Systems Engineering of Monash University, Melbourne, Australia (e-mail: ahmet.sekercioglu@monash.edu). features extraction from time segmented transient and steady-state vibrations contents. Several feature extraction methods based on time, frequency and multi resolution analysis (MRA) [1]-[10] have been used for fault patterns classification. FT based techniques provide translation invariant spectral features and are among the most widely used feature extraction tool for MHM [1]-[5]. One of the major concerns of FT based techniques is that the accuracy of extracting constituent transient frequencies amplitudes information is limited by the length of the segmentation window relative to the duration of the transient signature [7]. In FT small duration transients, in time domain, are averaged over the entire segmentation window length to transform them into frequency domain and results in belittled spectral amplitudes calculation. Further, FT based methods perform poorly to locate transients on the time axis. To subdue this deficiency, MRA (wavelet analysis (WA)) is used. In WA, vibration signals are decomposed into several nodes, each node covers a specific frequency sub band [6], [7]. Thus, most of the transients and steady-state vibration contents fall in different nodes and are discernible for augmented amplitude calculation for classification. WA also provides high resolution, both, in time and frequency domains. But, wavelets are inherently translation-variant, which makes WT nodes contents time-moving and thus, vibration signal contents keep on changing their location in time domain window in their respective nodes. Using these contentsfor fault pattern classification often turns out to be tedious or leads to lesser accurate results [9]. To ease with fault classification with WA, statistical features from these nodes are calculated for classification purposes [6], [9]. These statistical features discard most of the time and multi-resolution information; and contain only a fraction of total information. In contrast, FT has the advantage of representing time moving vibration signals in the form of non-moving, translation invariant spectral features. These translation invariant patterns can be processed and used as direct input to a classifier for training and, thus, statistical features calculation can be avoided. It is obvious that WT and FT complement each other in localizing transients in time, augmented transients amplitude calculation and translation invariant features extraction. Thus in this paper, strengths of WT and FT are cascaded to complement each other to extract more suited features which are to be fed to a classifier for fault classification. Thus, in this proposed method, time segmented vibrations are transformed to several nodes using WT. These nodes have translation variant but augmented information of transients and steady-state vibrations at different decomposition levels. These contents are then 
passed through FT to get translation invariant spectral feature set. This feature set is then fed to a suitable classifier for training and then testing purpose.

ANNs, SVM, Fuzzy classifier, K-means clusters, Bayesian algorithms and statistical methods are among widely used classifier for MHM [2]-[5], [9], [11]-[21] in literature. Selection of an appropriate classifier depends upon diagnosis type, features dimensionality, and available training data sets.

With cascaded WT and FT we have spectral contents over several decomposition levels and thus results in high dimensional features. ANNs are known for their capabilities to learn high dimensional features with non-linear and complex patterns and have been used for many MHM techniques of prognosis, classification, function approximation, control filter and pattern recognition [2]-[5], [8], [9], [11], [12], [14], [15]. Because of high dimensionality of features and supporting translation invariant features, ANN has been used as a classifier in this paper. Results have shown that proposed solution of ANN classifier, based on cascaded WT and FT features, has surpassed existing solutions in classification accuracy and robustness. Fig. 1 shows the framework of the proposed method.

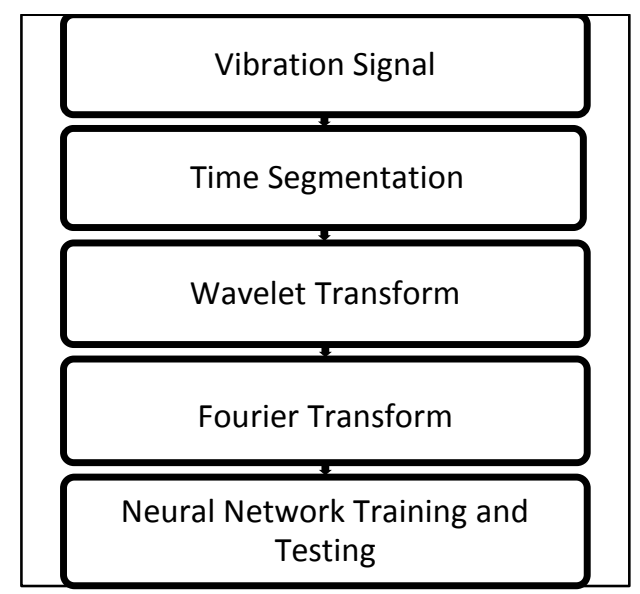

Fig. 1. Framework of the proposed method.

The remaining paper is organized as follows: Section II describes time segmentation of the vibration signal, Section III presents WT and FT based spectral features set extraction, Section IV introduces ANN architecture and training, Section $\mathrm{V}$ discusses experimental results and Section VI, finally, concludes the paper.

\section{TIME SEGMENTATION}

Continuously recorded vibration signal is, first divided into several time segments using a fixed rectangular window [10]. The diagnostic procedures are then applied to each of these segments. Let, $m$ be the total number of training or test set vibration instances obtained with a window size of $W$ samples for a signal $X$ of length $l$

$$
\boldsymbol{m}=l / w
$$

with $W$ window size, from total $m$ instances, time-domain contents of any vibration instance $i$ of $X$ are represented by

$$
\boldsymbol{x}^{i}=\left\{\boldsymbol{X}_{\boldsymbol{i}}(\boldsymbol{w})\right\}
$$

where $i=1,2,3, \ldots, m$ and $w=1,2,3, \ldots, W$

These time domain vibration segments are then used for augmented and translation invariant spectral features extraction using WT and FT. The process is explained in the next section.

\section{WAVELET AND FOURIER TRANSFORMS}

Time segmented vibrations obtained in the previous section contain the information of machine health. The features representing nature of faults need to be extracted from these segments. In the proposed cascaded WT and FT method, first, WT is used to decompose these segments, containing transients and steady-state contents of time vibrations, into several frequency sub-bands using continuous wavelet transform (CWT) function. CWT uses a mother wavelet wl, maximum scale a to transform time segment $x^{i}$ into frequency sub-bands by computing coefficients $C$ by

$$
C^{i}=C W T\left(x^{i}, a, w l\right)
$$

where $C$ is a $a \times b$ matrix. Where $a$ is maximum scale level and $b$ is time. Coefficients $16 \times 2048$ calculated from a vibration segments using db5 mother wavelet are shown in Fig. 2. These coefficients contain transients and steady-state fault signatures, at several frequency sub-bands. These signatures have augmented amplitudes but these are translation-variant in their respective sub-band. To use these contents for ANN efficiently we need time-invariant features for better classification accuracy, therefore we will apply FT to these time variant coefficients at all levels to get translation-invariant spectral features

$$
f^{i}(s c)=F T\left(C^{i}(s c)\right)
$$

$s c$ is a scale level and its value goes maximum to $a$. After transforming all the contents of sub-bands into frequency domain, the obtained spectral features are normalized as

$$
\bar{f}^{i}(s c)=f^{i}(s c) / \max \left(f^{i}(s c)\right)
$$

These normalized features for each scale level are calculated and are then combined to form training or test set

$$
\bar{f}^{i}=\left\{\bar{f}^{i}(1), \bar{f}^{i}(2), \bar{f}^{i}(3), \ldots, \bar{f}^{i}(a)\right.
$$

Each time segmented vibration is passed through (3)-(6) to get $\mathrm{m}$ training set examples for training of ANN. Feature set for window length 2048 and maximum scale level of 16 using $\mathrm{db} 5$ mother wavelet for some training samples of a vibration signal are shown in the Fig. 3.

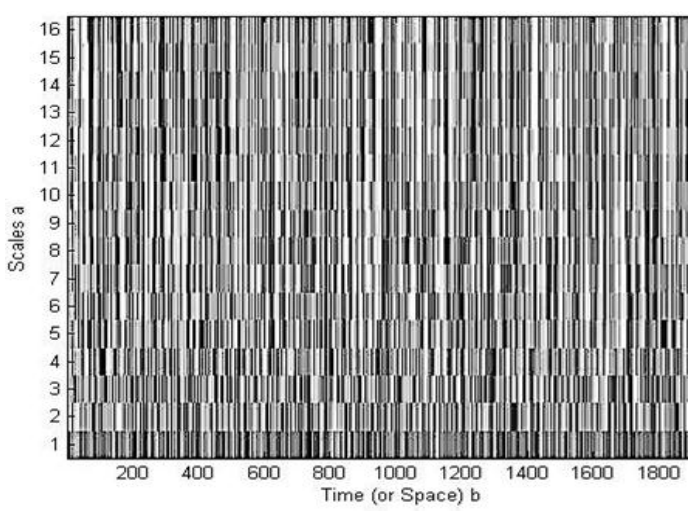

Fig. 2. Coefficients calculated by CWT function using db5 mother wavelet and maximum scale of 16 . 


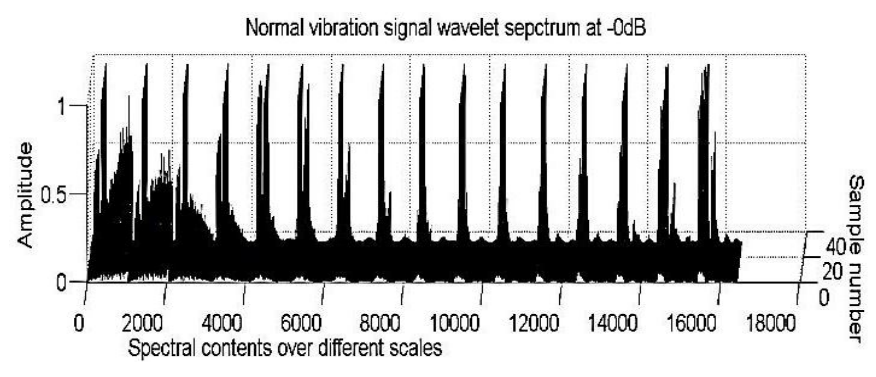

Fig. 3. Feature set of some vibration segments of normal machine behavior with window length of 2048 and scale levels up to 16 using db5 mother wavelet.

These spectral features are then used by ANN for training and diagnostic of the fault signals as discussed in the next section.

\section{ARTIFICIAL NEURAL NETWORK (ANN)}

ANN classifiers are biologically inspired nonlinear empirical models [5]. A typical ANN design includes an input layer, hidden layers and an output layer. An ANN learns different classes by tuning the weights between the connected neurons in different layers. Number of input layer neurons is equal to the input features and the number of output neurons is equal to number of classes. Between output and input layers there can be one or more hidden layers with different number of neurons. Minimum number of hidden layers and number of neurons giving the required classification accuracy are preferred to reduce computational complexity. ANN can be trained using supervised or unsupervised learning depending upon nature of the problem and available data. In this paper supervised learning has been used as for each input features pattern (6) we have an associated target output.

Before starting the training, the given data is split into training, validation and test tests. ANN is then trained by supervised learning using feed forward and back propagation algorithm. MSE is used to measure the learning of the training, testing and validation of ANN. The training of ANN using experimental dataset will be presented in the next section of experimental results and discussion.

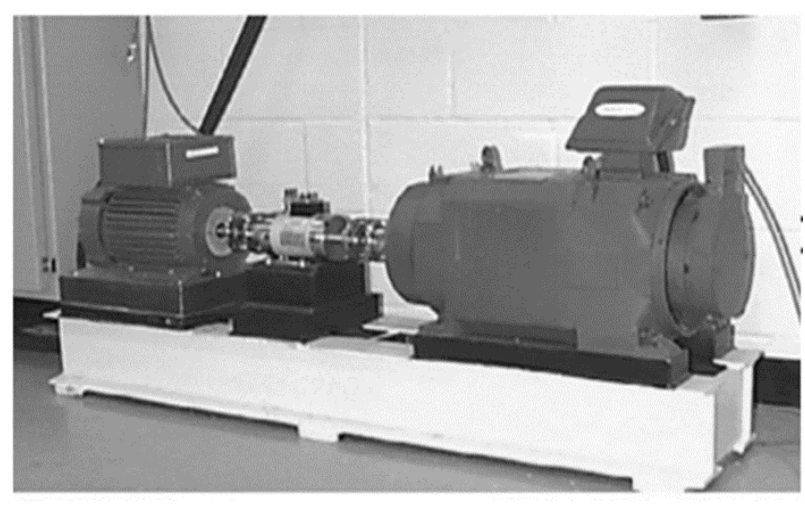

Fig. 4. Experimental setup adapted from [22].

\section{EXPERIMENTAL RESULTS AND DISCUSSION}

\section{A. Experimental Setup}

The proposed method has been tested with publically available dataset. The experimental setup for recording the vibration data sets is shown in the Fig. 4 and details are available in [22]. Data have been collected using four different bearings including one normal and three having faults in their inner race, ball and outer race. Electro-discharge machining has been used for fault generation in the bearings of several intensities and these faulty bearings support the shaft of the motor with a load of 2 HP. The vibration data of the machine, running at $1750 \mathrm{rpm}$, has been collected through accelerometers using a 16-channel digital-audio-tape recorder and sampled at the rate of 12000 samples per second.

The data collected from above experimental set up has been converted into time segmented vibrations with a window size of 2048 samples using (1) and (2). These, time segmented, vibrations were passed through the process explained in (3)-(6), using a maximum scale level of 16 and db5 mother wavelet, to get augmented and translation invariant spectral features training set for the ANN. To test the robustness of the proposed method, vibrations at several SNR levels need to be tested. Fig. 5 shows a spectral feature set of usual behavior of bearing over different sample numbers at SNR of $-10 \mathrm{~dB}$.

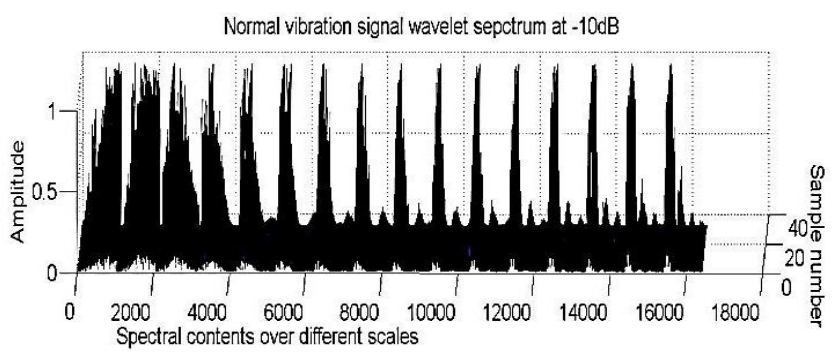

Fig. 5. Feature set of normal behavior vibration signal with window length of 2048 and scale levels up to 16 .

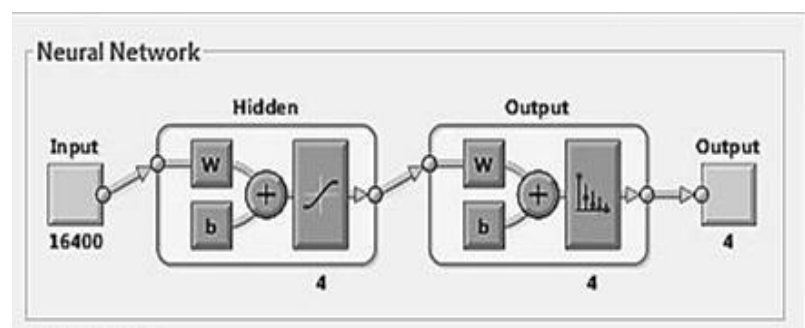

Fig. 6. Trained neural network architecture

Based on training set obtained, the selection of ANN architecture and its training are discussed next.

\section{B. ANN Training}

Feature set obtained from experimentally recorded dataset for four vibration classes was randomly shuffled and divided into $70 \%$ training, $15 \%$ validation and $15 \%$ testing set. Feed forward and back propagation algorithm has been used to train the ANN for different class features classification at SNR of 0dB. Each spectral feature set instance has 16400 features and are the inputs of the ANN. The four target classes; normal, inner race fault, outer race fault and ball faults, are the output of the ANN. ANN with one hidden layer, having 16400 inputs and four outputs, has been studied with different number of hidden layer neurons. Increasing the number of hidden layer neurons increases the computational cost. Thus, minimum number of hidden layer neurons is preferred provided they can achieve required classification accuracy. More than three neurons in the hidden layer gave 
the required accuracy. Thus four neurons, minimum number of neurons giving us required classification accuracy, in the hidden layer have been selected. The architecture of the selected ANN is shown in the Fig. 6.

The curves for training, validation and testing of the ANN are shown in the Fig. 7.

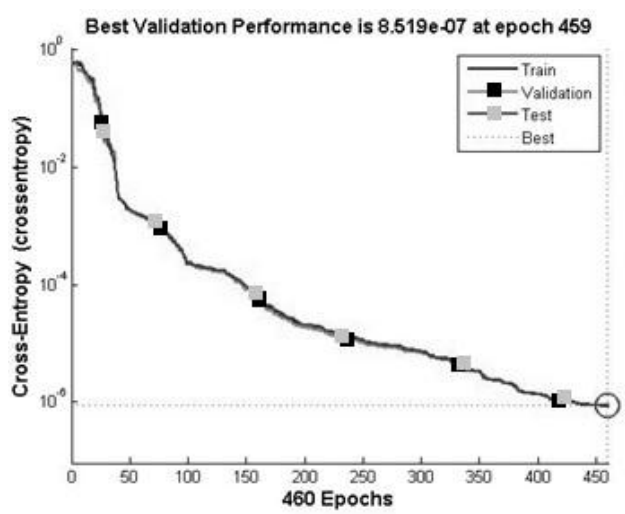

Fig. 7. Training curves of ANN.

ANN has achieved a Mean Square Error (MSE) of $8.519 \mathrm{e}^{-07}$ as shown by validation curve. After training, ANN was tested with different vibration signals from experimental setup with different faults and it was able to classify those vibrations into their respective classes with $100 \%$ accuracy with $\mathrm{SNR}=0 \mathrm{~dB}$. The classification accuracies of the ANN for test, train and validation set are shown in the Fig. 8 in the form of confusion matrix with percentages.
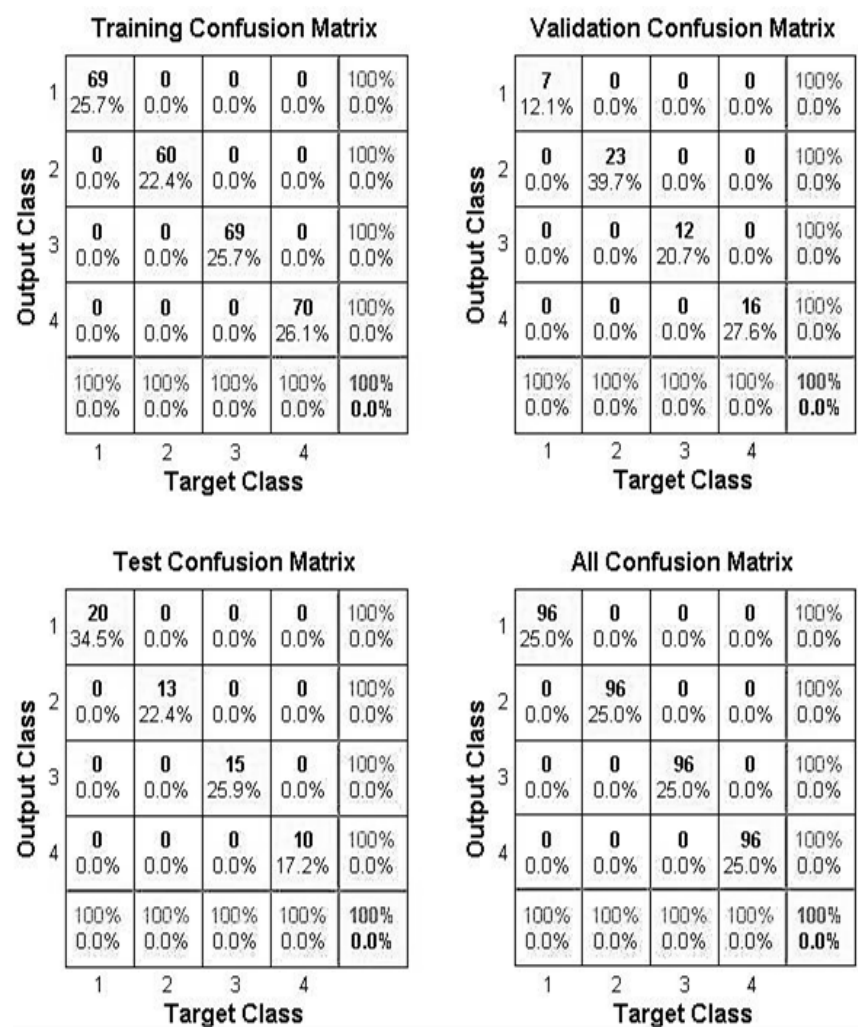

Fig. 8. Confusion matrix for training, testing and validation sets.

\section{Classification Accuracy and Robustness}

Now we will discuss the classification accuracy of the trained ANN under adverse SNR conditions to validate the robustness of the cascaded WT and FT features based ANN classifier. Fig. 9 shows the confusion matrix of cascaded WT and FT based ANN classifier at SNR of $-10 \mathrm{~dB}$. At this adverse SNR, proposed method still achieves $95.3 \%$ classification accuracy.

\begin{tabular}{|c|c|c|c|c|c|}
\hline & & & usion $\mathrm{A}$ & & \\
\hline 1 & $\begin{array}{c}\mathbf{9 6} \\
25.0 \%\end{array}$ & $\begin{array}{c}\mathbf{0} \\
0.0 \%\end{array}$ & $\begin{array}{l}\mathbf{3} \\
0.8 \%\end{array}$ & $\underset{0.0 \%}{\mathbf{0}}$ & $\begin{array}{l}97.0 \% \\
3.0 \%\end{array}$ \\
\hline 2 & $\begin{array}{c}\mathbf{0} \\
0.0 \%\end{array}$ & $\begin{array}{c}\mathbf{9 0} \\
23.4 \%\end{array}$ & $\begin{array}{c}\mathbf{3} \\
0.8 \%\end{array}$ & $\begin{array}{c}\mathbf{0} \\
0.0 \%\end{array}$ & $\begin{array}{l}96.8 \% \\
3.2 \%\end{array}$ \\
\hline & $\underset{0}{\mathbf{0}} \%$ & $\begin{array}{c}\mathbf{3} \\
0.8 \%\end{array}$ & $\begin{array}{c}\mathbf{8 4} \\
21.9 \%\end{array}$ & $\begin{array}{c}\mathbf{0} \\
0.0 \%\end{array}$ & $\begin{array}{c}96.6 \% \\
3.4 \%\end{array}$ \\
\hline 4 & $\begin{array}{c}\mathbf{0} \\
0.0 \%\end{array}$ & $\begin{array}{c}\mathbf{3} \\
0.8 \%\end{array}$ & $\begin{array}{c}\mathbf{6} \\
1.6 \%\end{array}$ & $\begin{array}{c}96 \\
25.0 \%\end{array}$ & $\begin{array}{r}91.4 \% \\
8.6 \%\end{array}$ \\
\hline & $\begin{array}{l}100 \% \\
0.0 \%\end{array}$ & $\begin{array}{l}93.8 \% \\
6.3 \%\end{array}$ & $\begin{array}{l}87.5 \% \\
12.5 \%\end{array}$ & $\begin{array}{l}100 \% \\
0.0 \%\end{array}$ & $\begin{array}{c}95.3 \% \\
4.7 \%\end{array}$ \\
\hline
\end{tabular}

Fig. 9. Confusion matrix of proposed classification tecchnique at $-10 \mathrm{~dB}$.

For comparison with the existing work, Table I shows the comparison of well-established techniques for classification accuracies mentioned in [6] with worst case scenario of $\mathrm{SNR}=-10 \mathrm{~dB}$.

TABLE I: CLASSIFICATION ACCURACY COMPARISON AT SNR $=-10$ DB [6]

\begin{tabular}{cc}
\hline \hline Scheme & \% Accuracy \\
\hline G. G. Yen [7] & 33.33 \\
X. Lou [18] & 33.33 \\
A. Malhi [19] & 38.09 \\
S. Seker [20] & 78.25 \\
F. Li [21] & 86.00 \\
M. F. Yaqub [6] & 91.23 \\
Cascaded WT and FT & 95.30 \\
\hline \hline
\end{tabular}

Table I shows that cascaded WT and FT based feature set and ANN classifier's accuracy surpasses the existing best case technique by $4.07 \%$ at $\mathrm{SNR}=-10 \mathrm{~dB}$. WT ability to augment and decompose transients and steady-state vibration signatures into several nodes, and time-invariant nature of FT spectral contents has helped to increase the classification accuracy even under low SNR. By increasing the scale level, the computational cost of the proposed techniques increases. Thus minimum number of scales is preferred, that is 16 in the proposed work to meet required classification accuracy. Though, this novel combination of cascaded WT and FT increases the computational cost but it helps in increased classification accuracy under low SNR. Further, to validate and generalize the improved robustness of the proposed method, results need to be compared using other datasets in future.

\section{CONCLUSION}

In this paper an ANN classifier using cascaded WT and FT based features for bearing fault classification has been presented. Time segmented vibrations have been decomposed to several frequency sub-bands using WT to achieve augmented transients signatures which are then converted to spectral features using FT to get translation invariant features. These enhanced transients and steady-state 
signatures amplitudes and translation invariant features, then, are used to train ANN classifier. Results have shown that ANN with cascaded WT and FT has achieved 95.30\% classification accuracy at worst case of $\mathrm{SNR}=-10 \mathrm{~dB}$. In future, minimum scales selection for WT will be studied to reduce the computational cost ensuring the required classification accuracy.

\section{REFERENCES}

[1] A. Bellini, F. Immovilli, R. Rubini, and C. Tassoni, "Diagnosis of bearing faults of induction machines by vibration or current signals: A critical comparison," in Proc. IEEE Ind. Appl. Society Annu. Meeting 2008, 2008, pp. 1-8.

[2] M. Amar, I. Gondal, and C. Wilson, "Multi-size-window spectral augmentation: Neural network bearing fault classifier," in Proc. The 8th IEEE Conf. Ind. Electron. Appl. ICIEA, 2013, pp. 261-266.

[3] B. Li, M.-Y. Chow, Y. Tipsuwan, and J. C. Hung, "Neural-network-based motor rolling bearing fault diagnosis," IEEE Trans. Ind. Electron., vol. 47, no. 5, pp. 1060-1069, Oct. 2000.

[4] S. Hayashi, T. Asakura, and S. Zhang, "Study of machine fault diagnosis system using neural networks," in Proc. Int. Joint Conf. Neural Netw., 2002, vol. 1, pp. 956-961.

[5] H. Su and K. T. Chong, "Induction machine condition monitoring using neural network modeling," IEEE Trans. Ind. Electron., vol. 54, no. 1, pp. 241-249, Feb. 2007.

[6] M. F. Yaqub, I. Gondal, and J. Kamruzzaman, "Inchoate fault detection framework: Adaptive selection of wavelet nodes and cumulant orders," IEEE Trans. Instrum. Meas., vol. 61, no. 3, pp. 685-695, Mar. 2012.

[7] G. G. Yen and K.-C. Lin, "Wavelet packet feature extraction for vibration monitoring," IEEE Trans. Ind. Electron., vol. 47, no. 3, pp. 650-667, June 2000 .

[8] Y. Yang and W. Tang, "Study of remote bearing fault diagnosis based on BP Neural Network combination," in Proc. 2011 7th Int. Conf. Natural Comput. (ICNC), July 2011, vol. 2, pp. 618-621.

[9] C. Chen, B. Zhang, and G. Vachtsevanos, "Prediction of machine health condition using neuro-fuzzy and bayesian algorithms," IEEE Trans. Instrum. Meas., vol. 61, no. 2, pp. 297-306, Feb. 2012.

[10] M. Amar, I. Gondal, and C. Willson, "Unitary anomaly detection for ubiquitous safety in machine health monitoring," in Proc. Neural Inf. Process., 2012, pp. 361-368.

[11] C. S. Tyagi. "A comparative study of SVM classifiers and artificial neural networks application for rolling element bearing fault diagnosis using wavelet transform preprocessing," in Proc. World Academy of Sci., Eng. Technol., 2008, vol. 33, pp. 319-327.

[12] J. F. Martins, V. F. Pires, and A. J. Pires, "Unsupervised neural-network-based algorithm for an on-line diagnosis of three-phase induction motor stator fault," IEEE Trans. Ind. Electron., vol. 54, no. 1 , pp. 259-264, Feb. 2007.

[13] F. Filippetti, G. Franceschini, C. Tassoni, and P. Vas, "Recent developments of induction motor drives fault diagnosis using Al techniques," IEEE Trans. Ind. Electron., vol. 47, no. 5, pp. 994-1004, Oct. 2000.

[14] C. Chen, B. Zhang, and G. Vachtsevanos, "Prediction of machine health condition using neuro-fuzzy and bayesian algorithms," IEEE Trans. Instrum. Meas., vol. 61, no. 2, pp. 297-306, Feb. 2012

[15] Y. Jun-rong, Y. MIN, C. Xia, and H. Yan, "Fault diagnosis of rolling bearing based on rough set and neural network," Appl. Mechanics Materials, vol. 58-60, pp. 974-977, 2011.

[16] M. Xia, F. Kong, and F. Hu, "An approach for bearing fault diagnosis based on PCA and multiple classifier fusion," in Proc. 6th IEEE Joint Int. Inf. Technol. Artificial Intell. Conf., 2011, pp. 321-325.

[17] C. T. Yiakopoulos, K. C. Gryllias, and I. A. Antoniadis, "Rolling element bearing fault detection in industrial environments based on a K-means clustering approach," Expert Syst. Appl., vol. 38, no. 3, pp. 2888-2911, Mar. 2011
[18] X. Lou and K. a Loparo, "Bearing fault diagnosis based on wavelet transform and fuzzy inference," Mech. Syst. Signal Process, vol. 18, no. 5, pp. 1077-1095, Sep. 2004.

[19] A. Malhi and R. X. Gao, "PCA-based feature selection scheme for machine defect classification," IEEE Trans. Instrum. Meas., vol. 53, no. 6, pp. 1517-1525, Dec. 2004

[20] S. Seker and E. Ayaz, "Feature extraction related to bearing damage in electric motors by wavelet analysis," J. Franklin Inst., vol. 340, no. 2 , pp. 125-134, Mar. 2003

[21] F. Li, G.Meng, L. Ye, and P. Chen, "Wavelet transform-based higher-order statistics for fault diagnosis in rolling element bearings," $J$. Vib. Control, vol. 14, no. 11, pp. 1691-1709, Nov. 2008.

[22] Bearing Data Center. (Jan. 2009). [Online]. Available: http://www.eecs. case.edu/laboratory/bearing/welcome_overview.htm

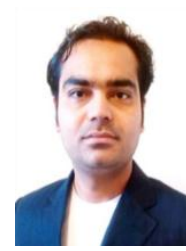

Muhammad Amar received his B.Sc. in electrical engineering from University of The Punjab Lahore in 2010 and M.Sc. degree in electrical engineering from University of Engineering and Technology, Lahore, Pakistan in 2013. He is currently a Ph.D. scholar in Monash University, Australia.

He has published over 25 peer-reviewed conference and journal publications. His research interests include the application of intelligent techniques in condition monitoring, diagnosis and monitoring, robotics, control systems and signal processing.

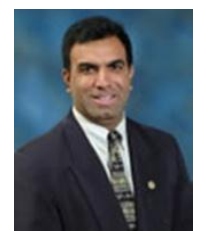

Iqbal Gondal received the B.Sc. degree in electrical engineering from University of Engineering and Technology, Lahore, Pakistan in 1990; and M.Sc. and Ph.D. degrees in electrical engineering from Victoria University of Technology, Australia in 1992 and 1996 respectively.

$\mathrm{He}$ has over 127 refereed conference and journal papers. According to Google Scholar, his publications have been cited over 622 times, with an H-index of 11 and G-index of 20 $\mathrm{He}$ has successfully supervised twelve and is currently supervising five $\mathrm{PhD}$ students.

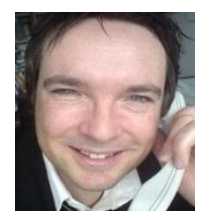

Campbell Wilson is a senior lecturer in the Caulfield School of Information Technology, Faculty of Information Technology Monash University, Australia. His research interests include information retrieval systems, machine learning and large scale data management.

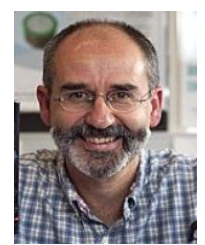

Ahmet Sekercioglu is a member of the academic staff at the Department of Electrical and Computer Systems Engineering of Monash University, Melbourne, Australia. He was the leader of the Applications Program of Australian Telecommunications CRC until the completion of the center's research activities in December 2007

$\mathrm{He}$ has completed his $\mathrm{Ph} . \mathrm{D}$. degree at Swinburne University of Technology, B.Sc. and M.Sc. degrees in electrical and electronics engineering at Middle East Technical University, Ankara, Turkey. He lectured at Swinburne University of Technology, Melbourne, Australia for 8 years. Prior to his academic career, he held numerous positions as a research engineer in private industry. He has published 22 journal articles, 2 book chapters, 71 conference papers and has filed 2 patents. His recent research is focused on distributed algorithms for self-organization in wireless sensor and ad hoc networks, and network robotics. 